\title{
An Empirical Model of Rotorcraft UAV Downwash for Disturbance Localization and Avoidance
}

\author{
Derrick Yeo ${ }^{1}$, Elena Shrestha ${ }^{2}$, Derek A. Paley ${ }^{3}$ \\ Department of Aerospace Engineering, University of Maryland, College Park, Maryland, 20742, U.S.A \\ and \\ Ella Atkins ${ }^{4}$ \\ Department of Aerospace Engineering, University of Michigan, Ann Arbor, Michigan, 48109, U.S.A
}

\begin{abstract}
Proximity operations of small rotary wing air vehicles pose a unique challenge due to the downwash they generate. These vertical flow disturbances may cause substantial and potentially hazardous flight disruption to nearby vehicles. This paper presents a downwash detection and localization strategy intended for use on small unmanned rotorcraft. A probabilistic framework estimates the position of neighboring rotorcraft operating at a higher altitude by combining onboard velocity measurements with a reduced-order flow model. A series of ground-based experiments that characterize the flow beneath a representative group of small rotary wing vehicles are described. A custom pressure probe designed to be carried by small rotary wing vehicles is mounted on an automated two-axis Cartesian carriage system, which enables accurate sensor placement. Velocity measurements collected in a prescribed path are assimilated into an axis-symmetric downwash model. Tests show that disturbances can be localized to the region directly beneath each vehicle while the sensor is still approaching, demonstrating basic downwash detection and localization capabilities for three different rotorcraft configurations.
\end{abstract}

$$
\begin{array}{ll}
u, v, w & =\text { Velocity components, } \mathrm{m} / \mathrm{s} \\
\rho & =\text { Air density, } \mathrm{kg} / \mathrm{m}^{3} \\
\mu & =\text { Dynamic viscosity of air, } \mathrm{kg} / \mathrm{ms} \\
v & =\text { Kinematic viscosity of air, } \mathrm{m} / \mathrm{s}^{2} \\
\beta & =\text { External source location vector } \\
z\left(t_{k}\right) & =\text { Flow measurement at time } t_{k}, \mathrm{~m} / \mathrm{s} \\
A & =\text { Posterior scaling coefficient } \\
r & =\text { Radial distance from central axis, } \mathrm{m} \\
W_{0} & =\text { Maximum profile velocity, } \mathrm{m} / \mathrm{s} \\
C & =\text { Velocity profile scaling factor }
\end{array}
$$

\section{Nomenclature}

\section{Introduction}

\footnotetext{
Qmall rotary-wing unmanned aircraft systems have recently seen widespread use throughout the public domain. Conventional helicopter configurations have been in service for a number of years [1,2] whereas multi-rotors have recently become synonymous with small unmanned aerial systems for recreational, research, and military applications [3,4]. With an increasingly crowded airspace, small air vehicles must face a new set of challenges associated with proximity flight. One specific challenge of rotary wing platforms is that their downwash can induce significant flow-field disturbances for other vehicles operating nearby and at a lower altitude. With minimal air traffic control regulations expected for small unmanned air vehicles (UAVs), onboard downwash detection could provide a means of disturbance avoidance on vehicles that have limited sensor capabilities.

${ }^{1}$ Research Associate, Aerospace Engineering Department, dyeo@umd.edu AIAA Member.

${ }^{2}$ Graduate Research Assistant, Aerospace Engineering Department, eshresco@umd.edu AIAA Student Member.

3 Willis H. Young Jr. Associate Professor, Aerospace Engineering Department and Institute for Systems Research, dpaley@umd.edu AIAA Senior Member.

${ }^{4}$ Associate Professor, Aerospace Engineering Department, ematkins@ umich.edu AIAA Associate Fellow
} 
This paper describes a downwash localization strategy that uses onboard air velocity measurements to provide disturbance detection and position estimates. Recursive Bayesian estimation is used in conjunction with a reducedorder flow model of rotorcraft downwash to assimilate vertical velocity component measurements and to generate probable locations of a nearby disturbance source. The approach is experimentally evaluated for three small UAVs with different rotor configurations (Fig. 1) using an automated two-axis Cartesian carriage system equipped with a custom pressure probe. The contributions of this research are (1) generalized downwash model for small rotorcraft UAVs developed for real-time estimation with limited onboard instrumentation; and (2) set of downwash surveys of three representative small rotorcraft UAV configurations. A companion paper illustrates the use of the downwash model for path planning [5].

The rest of this paper is organized as follows. Section II provides a background on small rotorcraft downwash characterization. Section III presents a probabilistic framework for sensing and avoiding downwash along with a reduced-order approach to downwash modelling. Section IV describes experiments that characterize the downwash beneath three different rotorcraft UAV configurations, which provide realistic flow fields to evaluate the localization strategy. Section V presents experimental data and Section VI documents localization results of the proposed estimation framework. Section VII summarizes the paper and describes ongoing work.

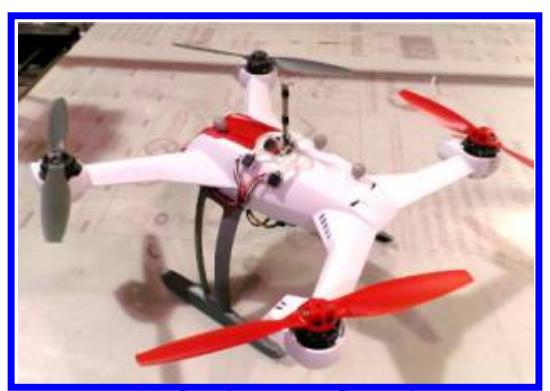

(a) Quadrotor configuration

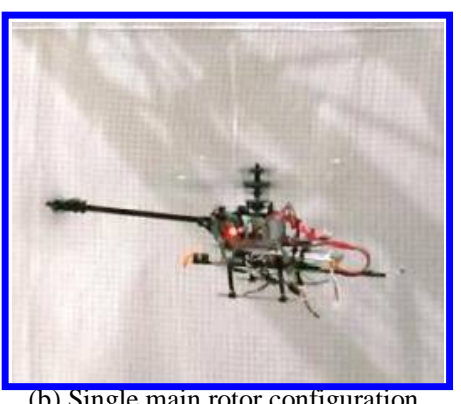

(b) Single main rotor configuration

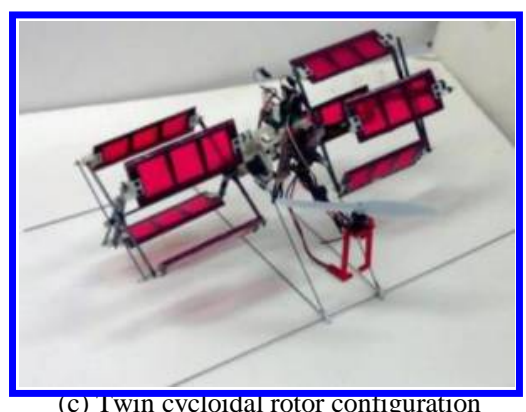

Figure 1: Small rotorcraft configurations currently in service at the University of Maryland

\section{Background}

Aerodynamic models that have previously been used to model conventional rotorcraft downwash [6] range from simple momentum theory to CFD models. Recent efforts in understanding rotor downwash effects on the environment have been motivated by desert environments commonly found in contemporary military operations [7, 8,9]. For example, Ghosh et al. [10] employed a CFD model to study the effect of rotor configuration on brownout, including a quadrotor configuration.

The more relevant field of small UAVs has also seen theoretical and experimental efforts. For example, Shkarayez et al. [11] conducted a series of wind tunnel tests that measured the contribution of propeller wash on the aerodynamic characteristics of a tail-sitter VTOL (vertical takeoff and landing) UAV concept, using force-balance instrumentation and hot-wire anemometry. Stone [12] modeled wing-propeller interaction on a tail-sitter UAV using a low-order fixed-wake panel method. These results have focused on wing-in-slipstream interactions, which are a secondary concern when attempting to detect and avoid a region of potentially hazardous downwash.

A low-order vortex model was employed by DeVries and Paley [13] to develop a sensing and estimation strategy to detect the wake of a refueling aircraft, providing a fixed-wing counterpart to the research proposed in this paper. Focusing on fundamental downwash characteristics, Khan et al. [14] developed a semi-empirical model that describes the wash generated by a single propeller. While directly relevant to small scale UAVs, the model is designed to simulate a single rotor using a set of known parameters, making it less suitable for a general, real-time estimation approach which must assume minimal prior knowledge of the flow field. Furthermore, it also does not account for the interaction that can be expected beneath a quadrotor. Yeo et al. [5] demonstrated the use of an even simpler model in conjunction with a Bayesian estimation scheme and an on-board flow sensing package for downwash disturbance on a small quadrotor UAV. Leese and Knight conducted a similar survey at full scale, using arrays of anemometers [15] to study the downwash of seven different military helicopters in service with the United States Army in 1974. A more recent test by Sjoholm et al. used a continuous-wave Doppler lidar to generate turbulent near-ground flow fields under a hovering rescue helicopter [16]. These flow measurements were taken near the ground. Small-scale experiments near the rotor have been conducted using hot-wire anemometry [17] and particle image velocimetry [18] techniques, while a custom designed multi-hole probe by Zilliac 19] has also 
successfully provided fine-scale wake-flow information [20]. Due to its portability and application to small scale UAVs, a probe-based instrumentation scheme is used in this work. This paper describes the development of this reduced-order approach to downwash modelling for real-time estimation onboard small rotorcraft UAVs. It documents a series of ground-based tests designed to evaluate its viability as a generalized model. A series of experimental surveys of the downwash generated by small rotary wing vehicles is included. An automated instrumentation positioning system has been built to take measurements along prescribed paths and the results from these surveys are used to evaluate the real-time estimation strategy.

\section{Localizing the Source of Rotorcraft Downwash}

This section presents a recursive estimation strategy that can detect downwash caused by a rotary-wing vehicle and generate estimates of its overhead location. This approach requires a reduced-order downwash model that is broadly applicable to a range of small rotary UAV configurations. It is theorized that fundamental flow physics result in common downwash features even for different rotorcraft configurations that can be estimated inflight using basic flow instrumentation. This section describes how insight from simple experimental data guided the derivation of simplified flow equations that are used within a Bayesian estimation scheme.

\section{A: Reduced-order modelling}

A flow-field model suitable for estimation needs to adequately capture key characteristics of rotorcraft downwash that is independent of configuration. In addition, it needs to function for wide variety of situations with little prior information. As all rotorcraft generate lift through momentum transfer with the air, this suggests a degree of self-similarity in their turbulent downwash that will be amenable to a generalized physical model. A two-bladed fan was chosen as the simplest representation of the downwash from a hovering rotorcraft. Axial velocity measurements taken within the zone of flow establishment were considered. Three key characteristics can be identified. The measured velocity near the center of the fan is higher than at the edge. Secondly, approximately exponential decay in velocity away from the centerline is also apparent. (This reflects the fundamental property of momentum conservation in a fluid flow.) Lastly, there are two velocity peaks surrounding the fan hub which is a characteristic of the velocity distribution before the slipstream flow is fully established.

These observations are qualitatively consistent with propeller slipstream measurements published in literature [14]. Within the zone of flow establishment, flow features near the centerline can be thought of as vehicle specific. Far below the vehicle and near the slipstream boundary, it is expected that the effects of diffusion will dominate. Based on this intuition, a theoretical middle ground between a purely physics-based approach and a first-order model is obtained through simplification of the Navier-Stokes equation. Consider a two-dimensional flow field where a rotor generates thrust by accelerating flow in the $z$ direction. Velocity components $w$ and $v$ lie along the $z$ and $y$ coordinates. The momentum equation in the $z$ direction is [21]

$$
\frac{\partial w}{\partial t}+w \frac{\partial w}{\partial z}+v \frac{\partial w}{\partial y}=g_{z}-\frac{1}{\rho} \frac{\partial p}{\partial z}+\frac{\mu}{\rho}\left(\frac{\partial^{2} w}{\partial z^{2}}+\frac{\partial^{2} w}{\partial y^{2}}\right)
$$

where $\rho$ is air density, $\mu$ is the dynamic viscosity of air, and $g_{z}$ represents body forces due to effects such as buoyancy.

The following set of assumptions are applied: (1) the mean flow-field is unchanging, so $\frac{\partial w}{\partial t}$ is zero; although rotorwash is highly turbulent, we consider the mean flow-field velocity generated by a hovering rotorcraft to be steady for these purposes. (2) Cross-stream flow is small compared to the downstream flow; this assumption is based on the intuition that even with the helical structure common to rotor-driven flows, the cross-stream velocity plays a secondary role in thrust generation. Only the thrust-aligned velocity component is considered and thus $\frac{\partial w}{\partial y}$ is neglected (3) At each downstream location, the stream-wise variation in $w$ is considered to be small compared to cross-stream changes; hence $\frac{\partial^{2} w}{\partial z^{2}}$ is ignored. (4) Buoyancy and external pressure gradients are considered to be absent, hence $g_{z}$ and $\frac{\partial p}{\partial z}$ are neglected. These assumptions result in

$$
w \frac{\partial w}{\partial x}=\frac{\mu}{\rho}\left(\frac{\partial^{2} w}{\partial y^{2}}\right)
$$


Solving (2) in cylindrical coordinates yields a Gaussian velocity profile that resembles observations of self-similar turbulent flows such as plumes and jets [22].

$$
w(r)=\frac{C}{z} W_{0} \exp \left(-\frac{W_{0} r^{2}}{4 z v}\right)
$$

Velocity profiles from experimental data are compared with the model in Fig. 2 . Having a Gaussian profile, the reduced-order model captures the exponential decay of velocity from the center line to the boundary of the downwash and the diffusion effects near the edges.

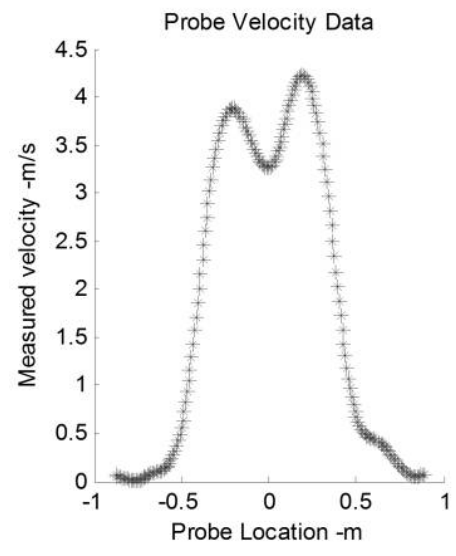

(a)

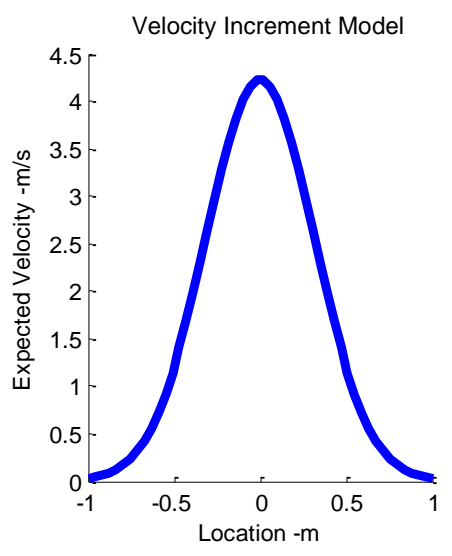

(b)

Figure 2: (a) Fan flow data and (b) simplified representation for estimation

This analysis leaves the parameters $C, z$ and $U_{0}$ to be determined. For the following analysis, these parameters were chosen based on fan experimental data. Ongoing work is focused on adaptive methods for choosing these parameters.

\section{B. Bayesian Estimation}

Recursive Bayesian estimation provides a probabilistic framework for inferring an unknown quantity given a set of measurements. The recursive Bayesian estimator used below is formulated as in [23]. It continuously updates the most likely location of an external source of downwash based on a set of vertical flow measurements. The location of the disturbance source is denoted by $\beta=\left(x_{s}, y_{s}\right)$ where $\left(x_{s}, y_{s}\right)$ are the coordinates of the disturbance center. Let $z\left(t_{k}\right)$ be a noisy wind speed measurement taken using the airspeed probe at time $t_{k}$. Given a set of measurements taken up until $t_{k}$, the probability of the vertical flow disturbance being centrally located at $\beta$ is [23]

$$
p\left(\beta \mid z\left(t_{k}\right)\right)=A p\left(z\left(t_{k}\right) \mid \beta\right) p\left(\beta \mid z\left(t_{1}, t_{2} \ldots . t_{k-1}\right)\right) .
$$

The mode of Eq. (4) is the maximum likelihood estimate, which denoted by $\hat{\beta}$. $A$ is a normalization constant to give the posterior unit integral. The measurement $z\left(t_{k}\right)$ is used to update the prior probability density $p\left(\beta \mid z\left(t_{1}, t_{2} \ldots t_{k-1}\right)\right)$ through the likelihood function $p\left(z\left(t_{k}\right) \mid \beta\right)$. The likelihood function is a conditional probability that represents the probability of receiving measurement $z\left(t_{k}\right)$ given a nearby vehicle located at $\beta$. The likelihood function is the key to implementing a Bayesian estimator as it translates raw measurements to information about the environment through prior understanding of the flow field. In this work, a Gaussian likelihood function is chosen to compute the probability of a measurement $z\left(t_{k}\right)$ by comparing a velocity measurement against a prediction provided by the flow model $w\left(r_{\beta}\right)$ in Eq. (3), where $r_{\beta}$ is the radial distance from the point of measurement and $\sigma^{2}$ is computed from the turbulent fluctuations measured.

$$
p\left(z\left(t_{k}\right) \mid \beta\right)=\exp \left(\left(z\left(t_{k}\right)-w\left(r_{\beta}\right)\right) / \sigma^{2}\right) .
$$




\section{Two-dimensional localization example}

The localization of a modelled downwash source was performed to validate the Bayesian estimation approach. The localization scheme is used with a single flow sensor approaching an idealized downwash source. The reducedorder model is used to generate both the estimated flow field and the sequence of sensor measurements. Uncertainty is introduced through mismatches in model parameters and through process noise that represents velocity fluctuations present in actual measurements. Results from four time steps are shown Fig. 4. The contour-maps depict the posterior distribution of the location of the disturbance. Red indicates high probability whereas blue indicates low probability. Red asterisks denote the measurement location. Fig. 4(d) also shows the path taken by the probe as it approaches the disturbance.

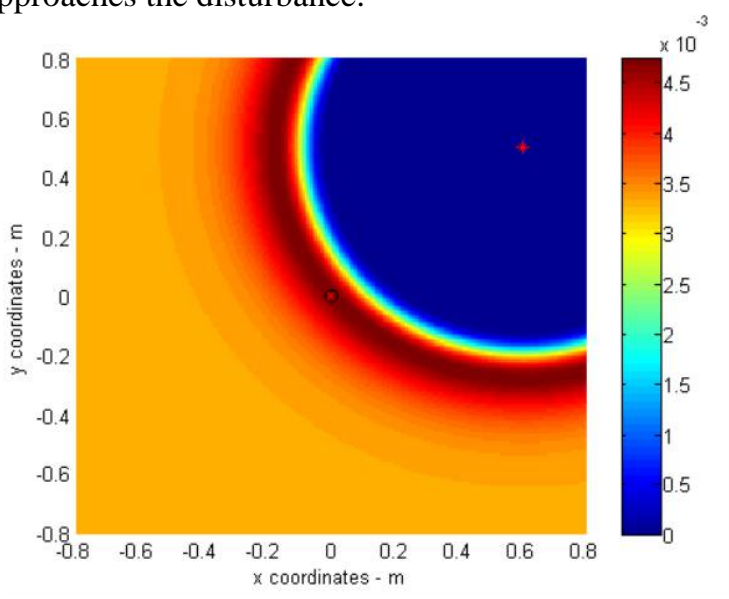

(a) $t=0$

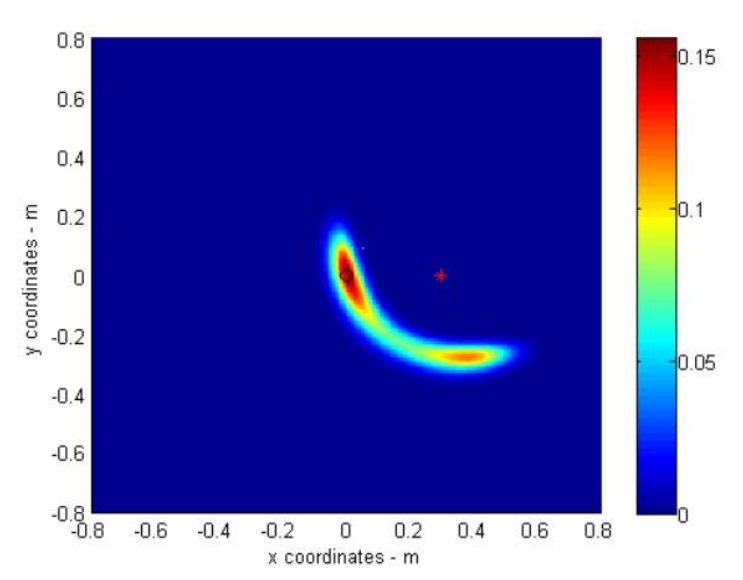

(c) $t=4$

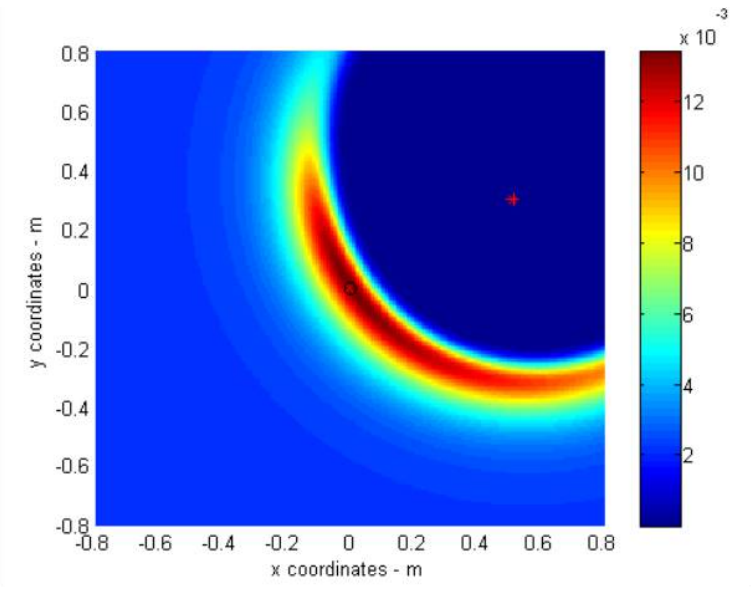

(b) $t=2$

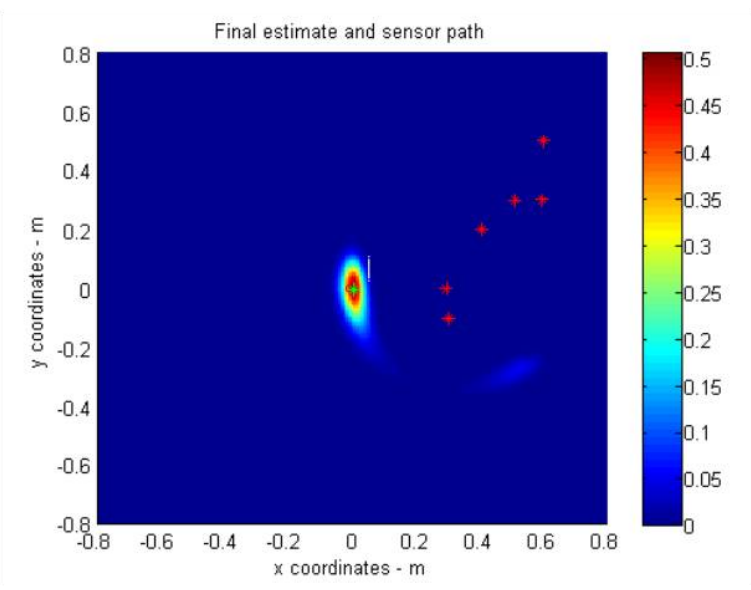

(d) $t=6$

Figure 3: Simulated localization results with numerically generated flow measurements

With no significant flow velocity measured initially, the estimation scheme reports a low probability of any downwash source within some radial distance of the sensor depending on the flow model and subject to the the measured turbulence level. As successive measurements are assimilated, arcs of high probability are computed, resulting in a final estimate that is close to the center of the idealized downwash. With a single sensor, the path taken by the sensor is critical as it is the only means to spatially distributing the measurements. For consistency, the same trajectory taken in this example is replicated using an automated instrumentation carriage system during experiments with actual downwash sources. These tests are described in the following sections. 


\section{Experimental Setup}

To evaluate the applicability of the estimation strategy to a range of small rotorcraft UAVs, a series of groundbased tests were designed to simulate flight trajectories of a sensor within the downwash of different rotorcraft configurations. The vehicles were suspended within the Collective Dynamics and Control Laboratory motioncapture facility to generate realistic flow fields. This section introduces the automated measurement system developed for these tests.

\section{A. Automated instrumentation carriage system}

A two-axis translating rail and carriage system was developed and built to support the flow measurements required for this work. A sliding carriage is driven over a guide rail with a servo-motor and drive wheel, providing translation in the inertial $x$-direction. The guide rail is itself propelled in the $y$-direction through a servo-motor powered winch and pulley system. The carriage system is controlled using position feedback from the motioncapture camera system. In addition to providing repeatable sensor positioning within a volume of interest, this system allows a sensor payload to be guided through prescribed trajectories in a plane. Experiments are coordinated by a desktop computer that communicates with the motion capture system, drive actuators, and flow instrumentation. A picture of the test setup and a breakdown of key components are shown in Fig. 4.

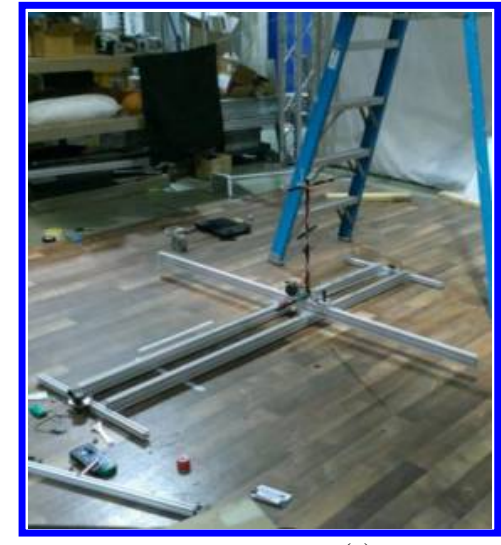

(a)

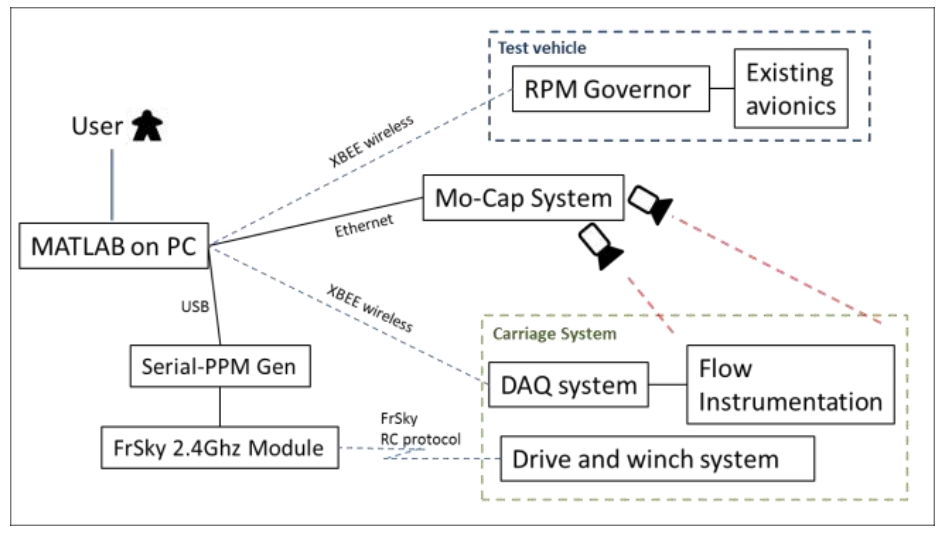

(b)

Figure 4: Automated instrumentation carriage and experimental system overview.

\section{B. Custom velocity probe}

For this work, a pressure-based velocity probe [24] was mounted face- up and used to take flow velocity measurements within the downwash regions. Having been designed to be carried on a small UAV, it represents the sensor capabilities that can be expected of a small rotary wing vehicle [5]. While the probe can provide flow angularity information, only vertical velocity measurements are considered in this work. The probe and the mounting system are shown in Fig. 5.
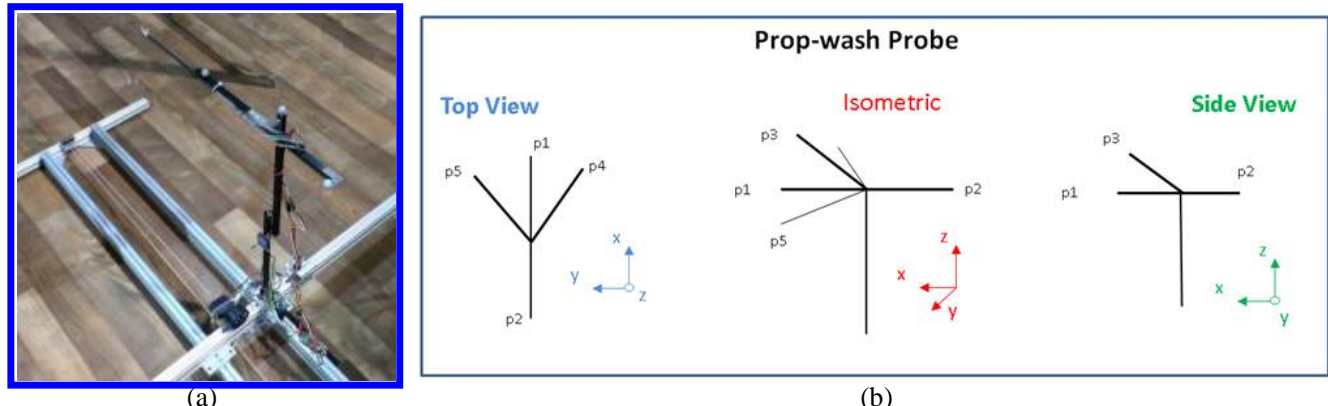

(b)

Figure 5: Multi-hole probe stand and port configuration [24] 
A MK20DX256VLH7 ARM cortex M4 microprocessor was used for data acquisition, storage and transmission. Pressure measurements across the ports are taken using Honeywell HSCDRRN series differential pressure sensors at $1 \mathrm{kHz}$ for 5 seconds with 13bit Analog-to-digital-convertor resolution.

\section{Small UAV downwash measurements}

Downwash measurements from beneath multiple small rotorcraft UAVs were sought to experimentally evaluate the proposed estimation strategy. This section presents velocity measurements from a series of flow surveys conducted on a two-bladed propeller and three different rotary UAV configurations. In each case, the outline of the rotors are super-imposed over the velocity contours in black and green lines. The axes are aligned with body frame, providing a top-down view of the vehicle as it faces the right side of the page. The avionics package on each test vehicle is augmented with a Hall-effect sensor and embedded electronics to govern rotor RPM.

\section{A. Isolated propeller}

A two bladed, 13x6 propeller was used to provide a simple, axisymmetric flow field. Since the flow generated would be similar to the modelled downwash, this reference case would provide insight on the effects of flow turbulence on the estimation strategy. The propeller was rotated at 3500 RPM, corresponding to a tip Reynolds number of approximately 70,000. The velocity contours at four diameters downstream are shown in Fig. 6.

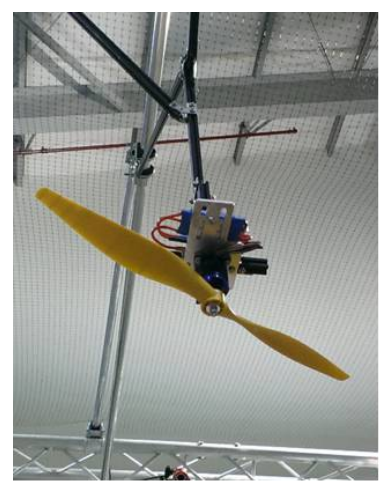

(a) Mounted test article

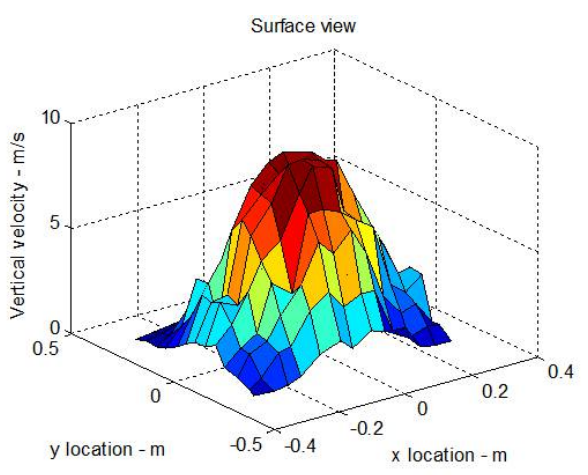

(b) Velocity surface

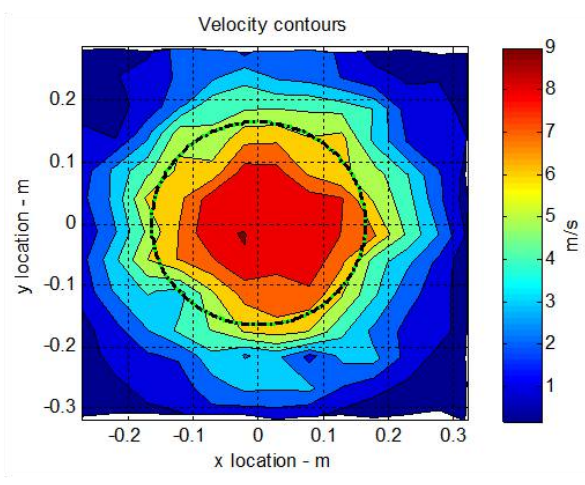

(c) Velocity Contours

Figure 6: Isolated propeller test setup and velocity data at 4 diameters downstream

\section{B. Single main rotor}

The conventional configuration uses a single main rotor and a tail rotor for anti-torque. For this experiment, a Dynam 250 two-bladed radio control helicopter with a $500 \mathrm{~mm}$ rotor diameter was used to generate the flow field. The swashplate and tail rotor pitch control linkages were adjusted for a hover condition and secured using vibrationisolation rubber. The velocity profiles in Fig. 7 were taken at 3.5 diameters beneath the vehicle. It shows a largely axisymmetric distribution that is expected from a single main rotor.

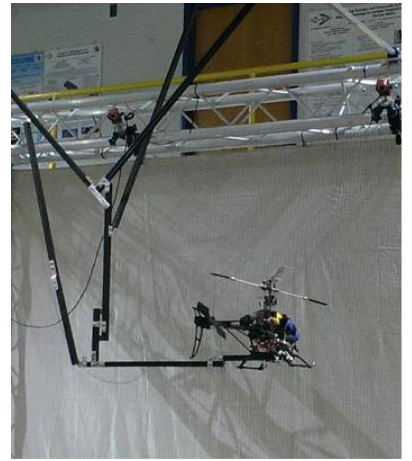

(a) Mounted test article

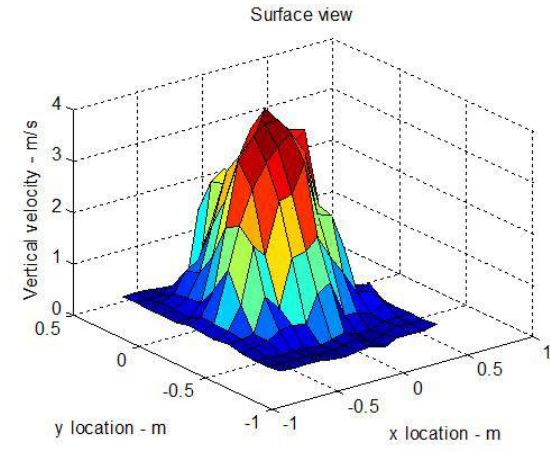

(b) Velocity surface

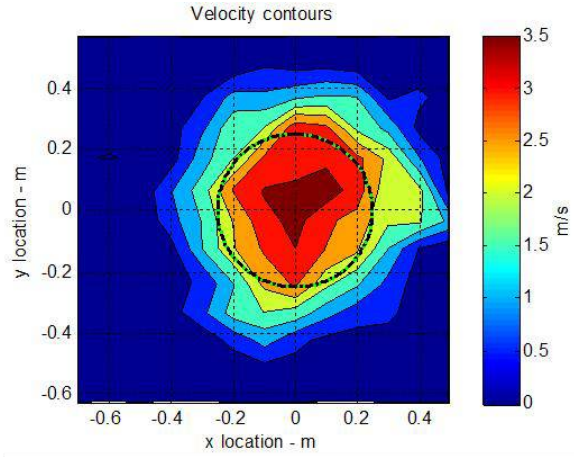

(c) Velocity Contours

Figure 7: Conventional helicopter velocity distribution at 3.5 rotor diameters 


\section{Quadrotor}

By using two pairs of counter-rotating propellers, the quadrotor helicopter configuration allows for a mechanically simple rotorcraft design with few moving parts. The vehicle used for these tests is a DJI Phantom, a small quadrotor that uses 8 -inch diameter propellers. The velocity distributions at 7 rotor diameters below the vehicle are shown in Fig. 8.

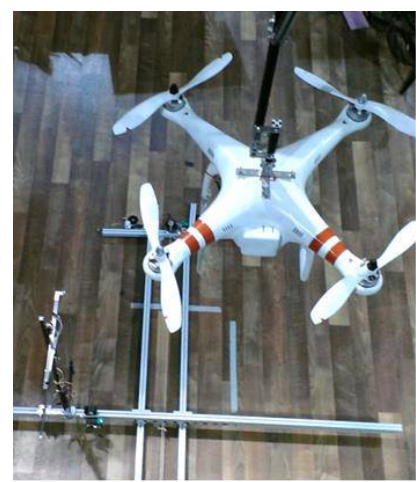

(a) Mounted test article

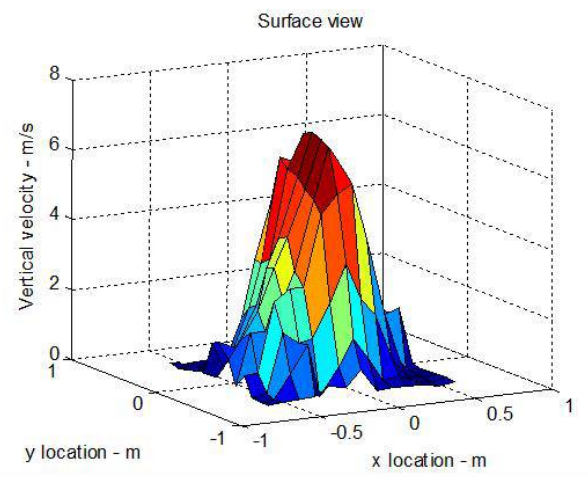

(b) Velocity surface

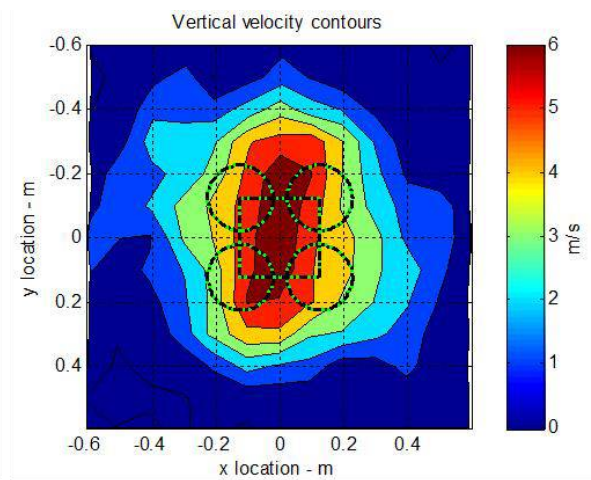

(c) Velocity Contours

Figure 8: Quadrotor vertical velocity profiles at 7 rotor diameters

It can be observed that within 5 rotor diameters, the vertical velocity had an approximately elliptical Gaussian distribution due to interaction between the wash of the two counter-rotating pairs of rotors. Each counter-rotating pair generates slipstreams that reinforce or weaken each other as they meet on along a plane of symmetry. With the Phantom's rotor configuration, the slipstreams reinforce each other along the plane of symmetry that extends out the sides of the vehicle, while mutually weakening along the plane that extends through the front and back of the vehicle. This effect is also observed with tandem and tilt-rotor helicopters [25]. With a single velocity peak, this profile is relatively simple but only approximately possesses rotational symmetry about a central axis.

\section{Cycloidal rotor}

Unlike most rotary wing vehicles, the UMD twin cyclocopter uses a pair of cycloidal rotors to generate lift and thrust while a third conventional propeller generates both lift and anti-torque in the pitch axis [26,27]. The 6 inch diameter cycloidal rotors accelerate flow downwards and towards the front of the vehicle (towards the propeller system) due to their rotation. The torque generated by the horizontal propeller is counteracted through an asymmetric thrust vector setting on the cycloidal rotors. This generates a vertical velocity distribution that is asymmetric front-to-back and side-to-side as shown in Fig. 9.

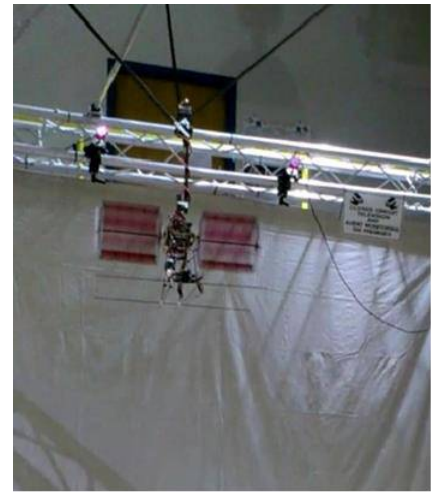

(a) Mounted test article

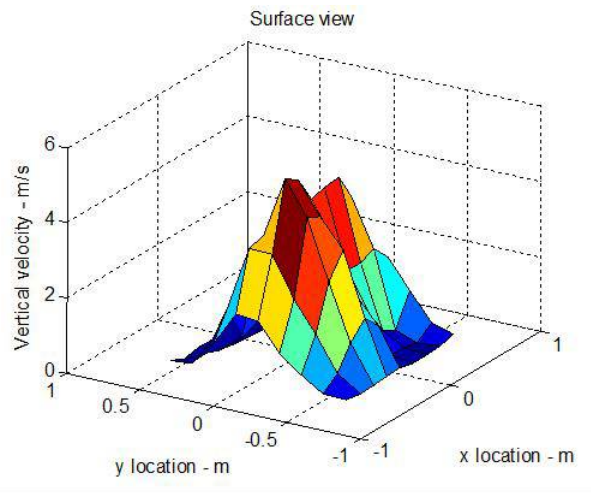

(b) Velocity surface

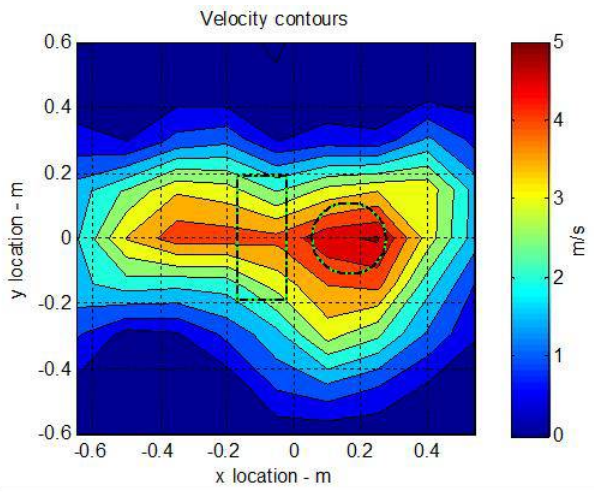

(c) Velocity Contours

Figure 9: Cyclocopter velocity profiles at 8 rotor diameters 


\section{Localization Results}

The experimental data from the downwash surveys was used to evaluate the estimation strategy in different realistic flow fields. These results are summarized in the following subsections. In each case, the probability density at the final time step is plotted with the location of the mode $\hat{\beta}$ marked by a green asterisk. The sequence of sensor measurements is marked with red asterisks, and the footprint of the vehicle is outlined in green. As in Section V, figure axes are aligned with the body frame of the test article, showing a view of the vehicle from the top with the nose of the vehicle facing the right side. The flow model parameters and sequence of sensor positions used in the numerical simulation are replicated in each scenario. The intent is to gain insight into how much prior information is required by the estimation scheme to accommodate different flow fields and vehicle configurations and to motivate further model development and evaluation methodologies.

\section{A. Single Propeller Slipstream}

The estimation framework was first tested within the flow generated by a single propeller. Although the isolated propeller is axisymmetric, the sensor path was recreated to approach the propeller from the front and aft as a firstorder verification of the system. These results are shown in Fig. 10(a) and Fig. 10(b) respectively. It can be noted that variations in measured velocity and varying levels of turbulence between both sets of measurements result in a slightly different final location estimates. On both paths, the central location of the propeller is localized to within $15 \%$ of propeller diameter.

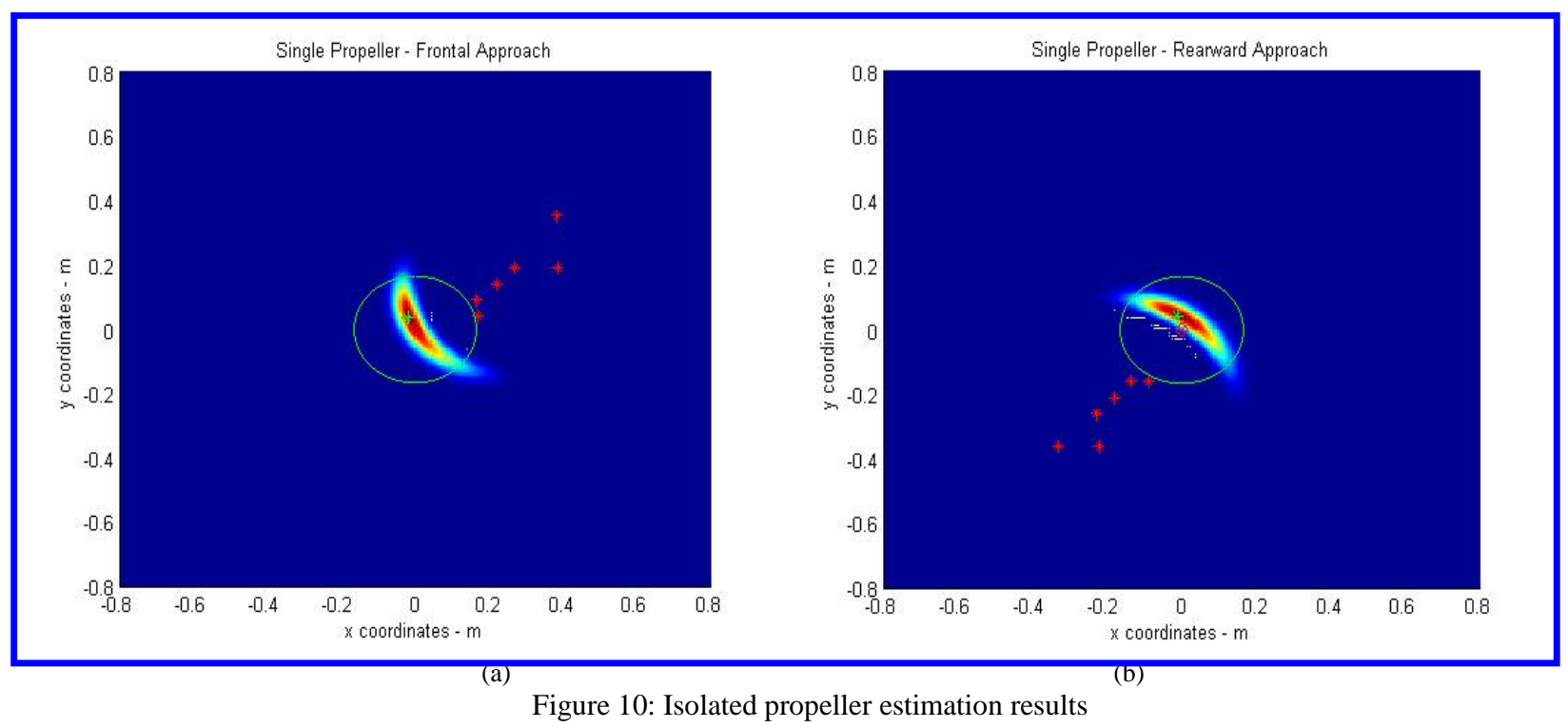

\section{B. Conventional Single-Main-Rotor Helicopter}

The flow field measured under the conventional helicopter in hover is similar to that generated by the single propeller. While the tail-rotor introduces a subtle left-to-right asymmetry on the downwash, this effect is not expected to significantly influence the estimation scheme. The sensor path used in the numerical simulation included both frontal and rearward vehicle approach in order to gauge the effect of the tail rotor in these tests. As expected, the performance of the localization scheme under a conventional helicopter is similar to that observed under a single propeller. For both trajectories, the center of the vehicle is localized to within $15 \%$ of main rotor diameter. The results are shown in Fig.11. 

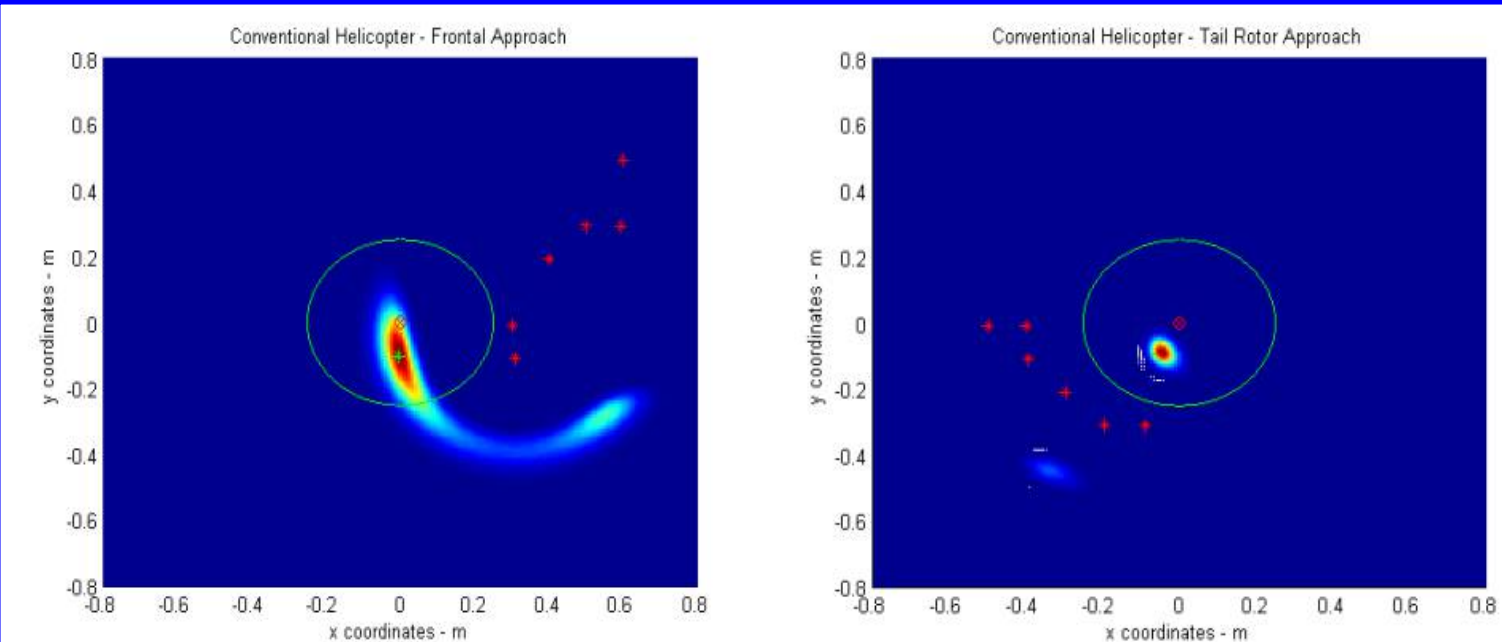

(a)

(b)

Figure 11: Estimation results for single-main-rotor configuration

\section{B. Quadrotor Helicopter}

The elliptical velocity profiles beneath the quadrotor offers an additional level of complication in the estimation scheme since the flow field is not axisymmetric. The velocity gradient recorded by a set of measurements depends on the orientation of the quadrotor. Two sensor paths are chosen that approach the vehicle between both planes of symmetry. Estimates were generated within 30\% of vehicle length and the localization results are shown in Fig.12. It is observed that these factors resulted in reduced localization accuracy compared to the conventional helicopter downwash case.

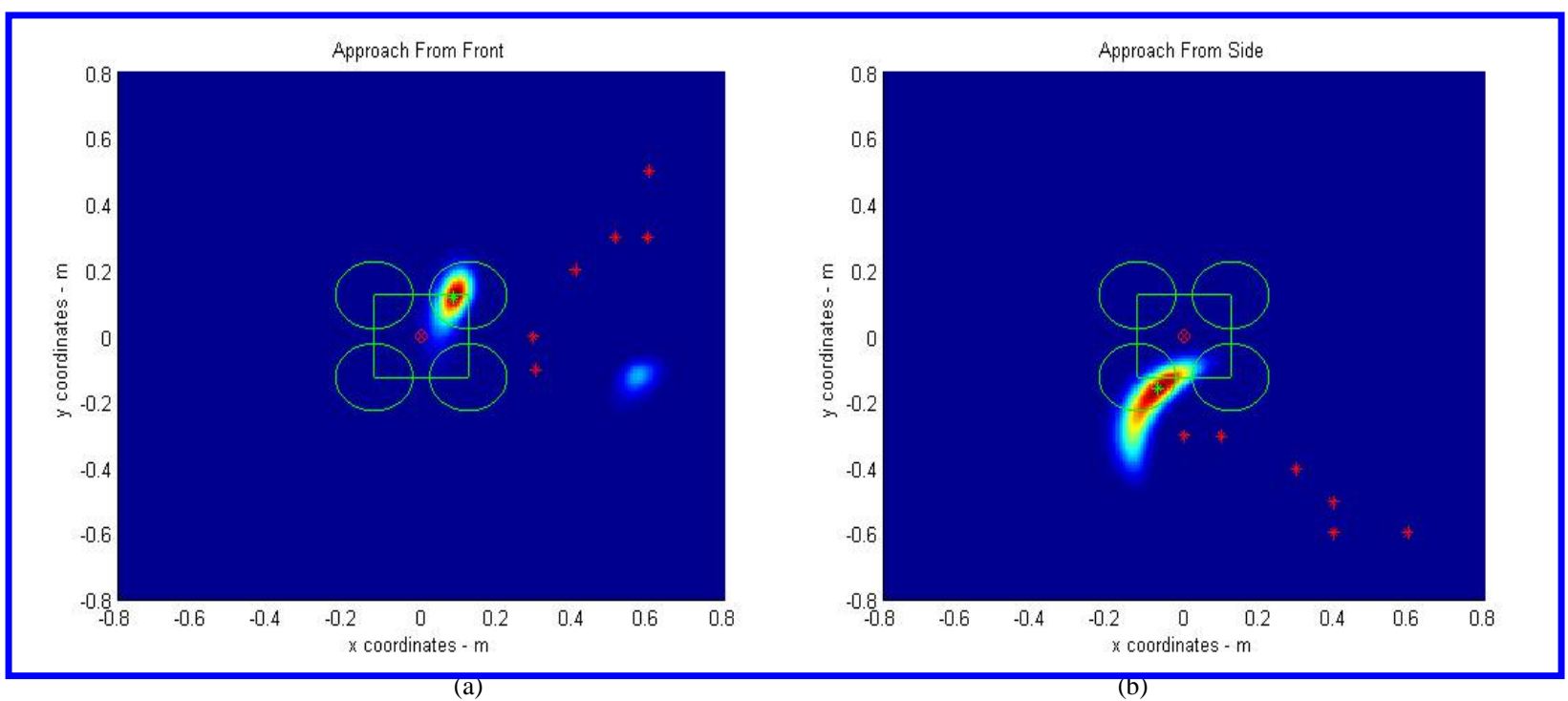

Figure 12: Quadrotor helicopter estimation results, front and side approaches

\section{C: Cycloidal Rotor Vehicle}

The flow-field of a hovering cyclocopter is more complicated than the previous configurations because of a distinctive fore-aft geometric asymmetry from the twin cycloidal rotors and a horizontal anti-torque rotor. These features are not represented in the flow model and may pose challenges to the estimation scheme. Four sensor paths were taken. The first set approaches the front and rear of the vehicle, where the conventional propeller is located. The second approaches the rear of the vehicle, where the cycloidal rotors and their unique velocity profiles are encountered. These results are shown in Fig. 13(a) and Fig. 13(b), respectively. 
The experiment was repeated with the same basic path approaching the vehicle from the sides, where the interaction between the cycloidal rotors and the horizontally mounted propeller create a profile expected to confound the estimation scheme. These results are presented in Fig 14.

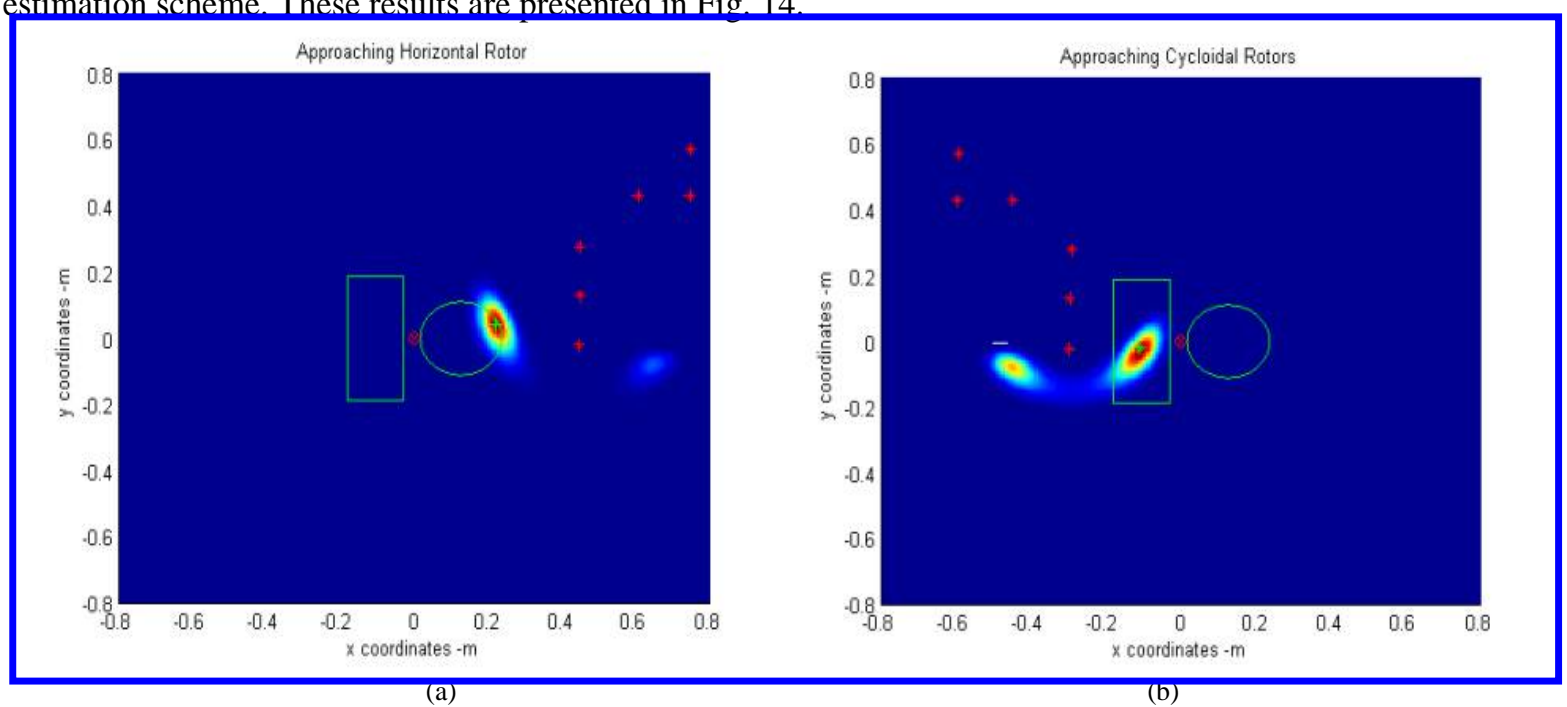

Figure 13: UMD twin cyclocopter localization results - fore/aft approaches

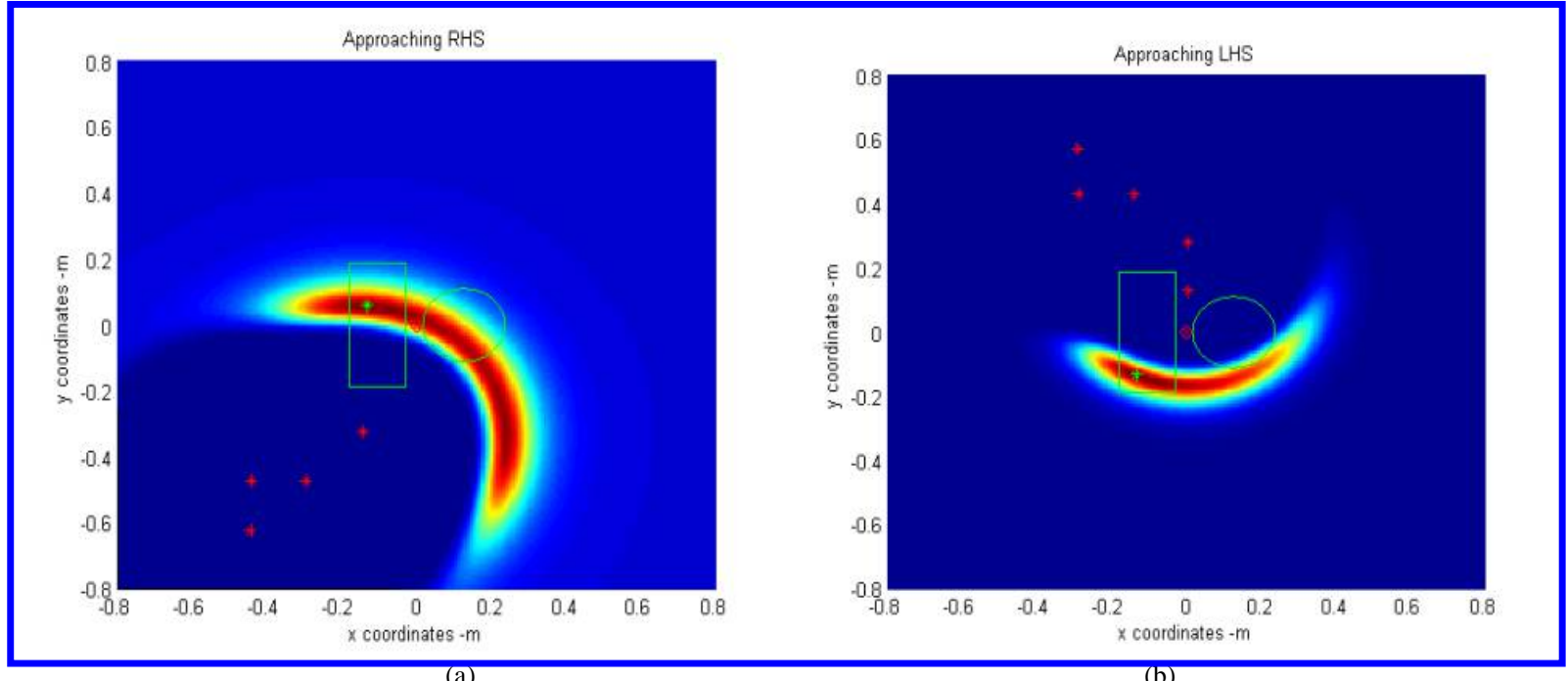

Figure 14: UMD twin-cyclocopter localization results - flanking approaches

The frontal and rearward approaches generate estimates that barely lie beneath the hovering vehicle. Observe that while the measurement paths from both directions generate feasible estimates, these flanking approaches have greater estimation uncertainty indicated by large arcs of probability. In all four cases, the localization scheme provides a final location estimate that is within the footprint of the vehicle, demonstrating a basic disturbance avoidance capability despite the simple flow model. These results reinforce the intuition that the increasing velocity gradients encountered on an approaching trajectory are a fundamental characteristic of any hovering rotorcraft and provide sufficient information to support simple disturbance avoidance schemes.

\section{D: Multi-Sensor Examples}

Multiple spatially distributed measurements at each time step enhances the accuracy of flow-field information. Two spatially distributed measurements are emulated by combining the data from two single sensor runs. Multiple- 
sensor runs for quadrotor and cyclocopter localization are shown in Fig. 15(a) and Fig. 15(b) respectively. In these plots, the locations of the second measurement are marked with a light blue asterisk.

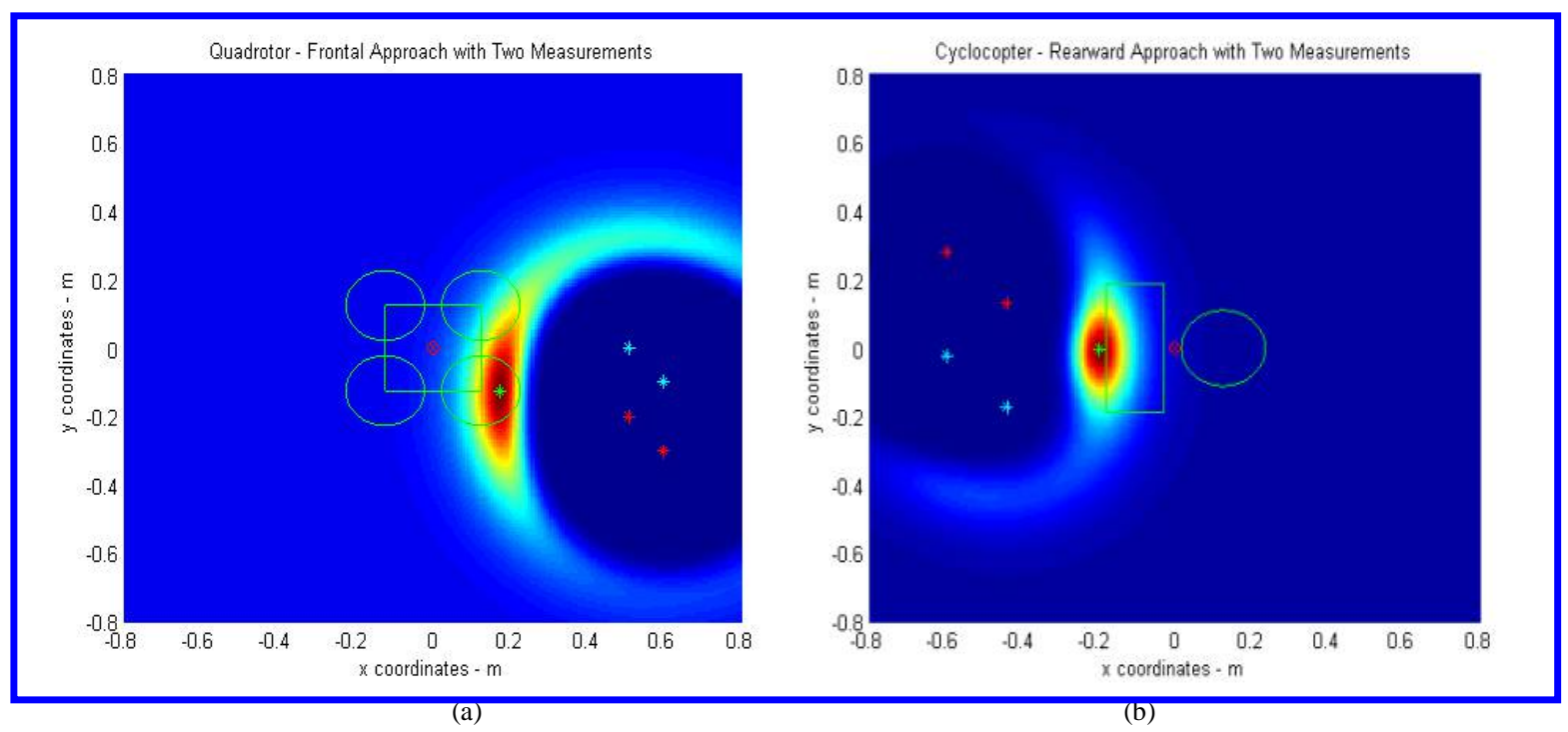

Figure 15: Preliminary two-sensor test cases

With spatially distributed measurements, more accurate estimates can be generated than from a single sensor. These results hint at the potential for comparable detection and localization capabilities at longer ranges from the disturbance source.

\section{Conclusions and Ongoing Work}

This paper describes a probabilistic framework that combines Bayesian estimation, onboard velocity measurements, and a simplified flow model for downwash detection and localization. To evaluate its applicability to different rotorcraft configurations, a series of flow surveys beneath three small rotorcraft UAVs were conducted to provide realistic flow fields. The approach is tested with a single flow sensor along a prescribed trajectory, using a simple axisymmetric flow model with fixed parameters. In this basic form, the system is able to generate useful position estimates for three different rotorcraft UAVs, localizing the disturbance source to the region directly below each vehicle during approach.

\section{Acknowledgements}

This material is based upon work supported by the U. S. Army under Grant No. W911W6112072 and the Air Force Office of Scientific Research under Grant No. FA95501310162.

\section{References}

$\checkmark 1 \quad$ E. Johnson, and S. Mishra. "Flight Simulation For The Development of An Experimental UAV"." Proc. AIAA Modelling and Simulation Technologies Conference and Exhibit, 5-8 August 2002, Monterey California. (AIAA 2002-4975)

$\triangleright^{2}$ M. Ligget. "RQ-8A Fire Scout VTUAV Environmental Control System Development." Proc. AIAA Joint Thermophysics and Heat Transfer Conference, 24-26 June 2002, St. Louis, Missouri. (AIAA 2002-3022)

3 M. Cutler, "Design and Control of an Autonomous Variable-Pitch Quadrotor Helicopter," . M.S. Dissertation, Department of Aeronautics and Astronautics, Massachusetts Institute of Technology, Cambridge, Massachusetts, 2012.

4 D. Snyder, "Design Requirements for Weaponizing Man-Portable UAS in Support of Counter-Sniper Operations," M.S. Dissertation, Department of Operational and Information Sciences, Naval Postgraduate School, Monterey, California, 2011. 
${ }^{5}$ D. Yeo, N. Sydney, D. Paley and D. Sofge. "Flow Sensing for Downwash Detection and Avoidance with Multiple Small Quadrotor Helicopters," To be presented at the AIAA Guidance Navigation and Control Conference, January 2015, Kissimmee, Florida.

6 J. Leishman. Principles of Helicopter Aerodynamics. Cambridge University Press, 2nd Ed. 2006.

$\checkmark$ B. Govindarajan, J. Leishman, and N. Gumerov, "Particle-Clustering Algorithms for the Prediction of Brownout Dust Clouds", AIAA Journal, Vol. 51, No. 5, pp. 1080-1094, 2013.

${ }^{8}$ C. Phillips and R. Brown. "Eulerian Simulation of the Fluid Dynamics of Helicopter Brownout", AIAA Journal of Aircraft Vol. 46 No.4, July-August 2009.

9 W. Polzin, K. Guntupalli, and G. Rajagopalan, "Discrete Blade Model for Rotorcraft Brownout", Proc. $28^{\text {th }}$ Applied Aerodynamics Conference, 27 - 30 June 2011, Honolulu Hawaii. (AIAA 2011-3182)

10 S. Ghosh, M. Kohry, and R. Rajagopalan. "Rotor Configurational Effect on Rotorcraft Brownout", Proc. $28^{\text {th }}$ Applied Aerodynamics Conference, 28 June- 1 July 2010, Chicago Illinois. (AIAA 2010-4238)

11 S. Shkarayev, J. Moschetta, and J. Bataille. "Aerodynamic Design of Micro Air Vehicles for Vertical Flight." AIAA Journal of Aircraft, Vol. 45, No. 5 , September-October 2008.

12 R. Stone. "Aerodynamic Modelling of the Wing-Propeller Interaction for a Tail-Sitter Unmanned Air Vehicle", AIAA Journal of Aircraft, Vol. 45. No. 1, January- February 2008.

13 L. DeVries and D. Paley. "Wake Estimation and Optimal Control for Autonomous Aircraft in Formation Flight," Proc. AIAA Guidance, Navigation and Control Conference, 19-22 August 2013, Boston Massachusetts. (AIAA 2013-4705)

14 W. Khan, R. Caverly and M. Nahon. "Propeller Slipstream Model for Small Unmanned Aerial Vehicles". Proc. AIAA Modelling and Simulation Conference, 19-22 August 2013, Boston Massachusetts. (AIAA 2013-4907)

15 G. Leese, and J. Knight. "Helicopter Downwash Data," U.S. Army Materiel Command Miscellaneous paper S74-17, U.S. Army Engineer Waterways Experiment Station Vicksburg, Mississippi AD 7807541974.

16 M. Sjoholm, N. Angelou, P. Hansen, K. Hansen, T. Mikkelsen, S. Haga, J. Silgerd and N. Starsmore. Helicopter Downwash Measured by Continuous-Wwave Doppler Lidars with Agile Beam Steering," Proc. $16^{\text {th }}$ International Symposium for The Advancement of Boundary-Layer Remote Sensing, 6-8 May 2012, Boulder, Colorado.

17 A. Wadcock, "Measurement of Vortex Strength and Core Diameter in the Wake of a Hovering Rotor," Proc. American Helicopter Society Technical Specialists meeting on Rotorcraft Acoustics and Aerodynamics, 28-30 October, 1997, Williamsburg, Virginia.

18 J. Heineck, G. Yamaguchi, A. Wadcock, L. Lourenco, "Application of Three-Component PIV to a Hovering Rotor Wake," Proc. $56^{\text {th }}$ American Helicopter Society Annual Forum, 2-4 May, 2000, Virginia Beach, Virginia.

19 G. Zilliac, "Calibration of Seven-Hole Pressure Probes for Use in Fluid Flows with Large Angularity," NASA Technical Memorandum 102200, Ames Research Center Moffett Field, California,December 1989.

20 J. Chow, G. Zilliac and P. Bradshaw, "Mean and Turbulence Measurements in the Near Field of a Wingtip Vortex," AIAA Journal, Vol. 35 No. 10, pp 1561-1567, October 1997.

21 F. White, "Viscous Fluid Flow", $3^{\text {rd }}$ edition, 2005,McGraw-Hill Engineering. 
22 R. Jensen, M. Albertson, Y. Dai and H. Rouse, “ Diffusion of Submerged Jets”. In Transactions of the American Society of Civil Engineers, Vol. 115, pp 639-697, 1950.

23 N. Gordon, D. Salmond and A. Smith. "Novel Approach to Nonlinear/non-Gaussian Bayesian State Estimation," IEE Radar and Signal Processing, Vol. 140, pp 107-113, 1993

24 D. Yeo, E. Atkins, L. Bernal and W. Shyy, "Aerodynamic Sensing for a Fixed Wing UAS Operating at High Angles of Attack," Proc. AIAA Atmospheric Flight Mechanics Conference, 13-16 August 2012, Minneapolis Minnesota.(AIAA 2012-4416)

25 J. Campbell, “National Aeronautics and Space Administration Research on Downwash Impingement”, Proc. VTOL Aircraft Downwash Impingement Symposium, U.S. Army Transportation Research Command, Fort Eustis, Virginia, 15 December 1960.

${ }^{26}$ M. Benedict, T. Jarugamilli, V. Lakshminarayan, and I. Chopra, "Experimental and Computational Studies to Understand the Role of Flow Curvature Effects on the Aerodynamic Performance of a MAV-Scale Cycloidal Rotor in Forward Flight," Proc. 53rd AIAA/ASME/ASCE/AHS/ASC Structures, Structural Dynamics, and Materials Conference, Honolulu, Hawaii, April 23-26, 2012.

27 E. Shrestha, M. Benedict, V. Hrishikeshavan, D. Yeo and I. Chopra, "Forward Flight of a Cycloidal Rotor MAV", Proc. $70^{\text {th }}$ American Helicopter Society International Forum, May 2014, Montreal, Quebec, Canada. 
This article has been cited by:

1. Derrick W. Yeo*, Nitin Sydney† and Derek A. Paley $\ddagger$ University of Maryland, College Park, Maryland 20742Donald Sofge§Naval Research Laboratory, Washington, D.C. 20375. 2017. Downwash Detection and Avoidance with Small Quadrotor Helicopters. Journal of Guidance, Control, and Dynamics 40:3, 692-701. [Citation] [Full Text] [PDF] [PDF Plus]

2. Richard B. Bramlette, Taylor A. Johnston, Ronald M. Barrett-GonzalezDesign, Construction, and Flight Testing of the World's Fastest Micro-Scale Quadcopter . [Citation] [PDF] [PDF Plus] 